\title{
THE CUT LOCUS OF A TORUS OF REVOLUTION*
}

\author{
JENS GRAVESEN ${ }^{\dagger}$, STEEN MARKVORSEN $^{\dagger}$, ROBERT SINCLAIR $^{\ddagger}$, AND \\ MINORU TANAKA ${ }^{\S}$
}

\begin{abstract}
We determine the structure of the cut locus of a class of tori of revolution, which includes the standard tori in 3-dimensional Euclidean space.
\end{abstract}

Key words. cut locus, a torus of revolution, geodesic

AMS subject classifications. Primary 53C20

1. Introduction. Let $(M, g)$ be a complete Riemannian manifold and $\gamma$ : $\left[0, t_{0}\right] \rightarrow M$ a minimal geodesic segment emanating from a point $p:=\gamma(0)$. The endpoint $\gamma\left(t_{0}\right)$ of the geodesic segment is called a cut point of $p$ along $\gamma$ if any extended geodesic segment $\widetilde{\gamma}:\left[0, t_{1}\right] \rightarrow M$ of $\gamma$, where $t_{1}>t_{0}$, is not a minimizing arc joining $p$ to $\tilde{\gamma}\left(t_{1}\right)$ anymore. The cut locus $C_{p}$ of the point $p$ is defined by the set of the cut points along all geodesic segments emanating from $p$. It is known that the cut locus of a point $p$ on a complete 2-dimensional Riemannian manifold is a local tree (see [5] or [13]), i.e., for any $q \in C_{p}$ and any neighborhood $U$ around $q$ in $M$, there exists an open neighborhood $V \subset U$ around $q$ such that any two points in $V \cap C_{p}$ can be joined by a unique rectifiable Jordan arc in $V \cap C_{p}$. Here a Jordan arc is an arc homeomorphic to the interval $[0,1]$. A standard torus in 3-dimensional Euclidean space defined by

$$
\left(\sqrt{x^{2}+y^{2}}-R\right)^{2}+z^{2}=r^{2} \quad(R>r>0)
$$

has the following two properties.

(1) The surface is symmetric with respect to the $(x, y)$-plane.

(2) The Gaussian curvature of the surface is monotone decreasing from the point $(R+r, 0,0)$ to the point $(R-r, 0,0)$ along the meridian defined by $y=0$.

The monotonic decline of the Gaussian curvature along a meridian is a very useful property for determining the structure of the cut locus of the standard torus. In fact, the structure of the cut locus of a class of surfaces of revolution containing all 2-sheeted hyperboloids of revolution and paraboloids of revolution was determined by making use of the monotonic decline of the Gaussian curvature in [2] (see also [16]). Let $T^{2}:=\left(S^{1} \times S^{1}, d t^{2}+m(t)^{2} d \theta^{2}\right)$ denote a torus with warped product Riemannian metric $d t^{2}+m(t)^{2} d \theta^{2}$, where $d t^{2}$ and $d \theta^{2}$ denote the Riemannian metric of a circle with length $2 a>0,2 b>0$ respectively and $m$ denotes a positive-valued $C^{\infty}$ warping function on $\mathbf{R}$ satisfying the following two properties.

$$
m(-t)=m(t)=m(t+2 a) \text { for any real number } t
$$

\footnotetext{
*Received May 20, 2003; accepted for publication June 28, 2004.

$\dagger$ Department of Mathematics, Matematiktorvet, Building 303, Technical University of Denmark, DK-2800 Kgs. Lyngby, Denmark (J.Gravesen@mat.dtu.dk; S.Markvorsen@mat.dtu.dk).

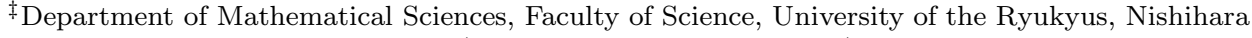
City, Okinawa Pref., 903-0213, Japan (sinclair@math.u-ryukyu.ac.jp).

$\S$ Department of Mathematics, Tokai University, Hiratsuka City, Kanagawa Pref., 259-1292 Japan (m-tanaka@sm.u-tokai.ac.jp). The work of this author was partially supported by the Grant-in-Aid General Scientific Research, No. 14540091.
} 


$$
\frac{m^{\prime \prime}(t)}{m(t)} \text { is monotone non-increasing on }[0, a]
$$

The Gaussian curvature $K$ at a point $q$ of $T^{2}$ is given by

$$
K(q)=-\frac{m^{\prime \prime}(t(q))}{m(t(q))}
$$

Thus by (1.3), the Gaussian curvature is monotone non-decreasing along a meridian from the point on $t=0$ to the point on $t=a$. If we define $m(t)=R-r \cos \frac{t}{r}, a=\pi r$, and $b=\pi$, then the torus $\left(S^{1} \times S^{1}, d t^{2}+m(t)^{2} d \theta^{2}\right)$ is isometric to the standard torus defined by (1.1). Thus the class of tori defined above contains the standard tori in 3-dimensional Euclidean space.

Our aim in this article is to determine the structure of the cut loci of a torus $\left(S^{1} \times S^{1}, d t^{2}+m(t)^{2} d \theta^{2}\right)$ of revolution, where $m$ is a positive-valued $C^{\infty}$-function satisfying (1.2) and (1.3). There are classical results on tori. In 1903, Bliss ([1]) investigated the behavior of geodesics on the standard torus and in 1930 Kimball ([8]) determined the conjugate locus of a point on a torus in 3-dimensional Euclidean space satisfying (1.2) and (1.3). The example constructed by Gluck and Singer ([3]) shows that one cannot impose any strong restriction on the structure of the cut locus of a point on a surface $S$, even if $S$ is assumed to be a surface of revolution with positive Gaussian curvature. The conditions (1.2) and (1.3) are thus reasonable and yet quite flexible in the sense that they are satisfied for a large family of tori of revolution, which contains all tori Kimball treated in [8]. By Theorems 3.2 and 4.1, which will be proved in this paper, we may state the structure of the cut loci of a standard torus defined by (1.1) as follows. The cut locus of a point $p=\left(x_{0}, 0, z_{0}\right)$ with $x_{0}>0$ on the torus is the union of

(i) the opposite meridian $y=0, x<0$,

(ii) a (piecewise $C^{1}$ ) Jordan curve which intersects the opposite meridian at a single point and is freely homotopic to each parallel,

(see Figure 1 in Section 5) and, if $p$ is sufficiently far from the inner equator, i.e., if $x_{0}>c_{2}$ for some positive constant $c_{2}(>R-r)$,

(iii) a pair of subarcs of the parallel $z=-z_{0}$, each with a conjugate point of $p$ as one endpoint and joining

- only the Jordan curve of (ii) if $c_{2}<x_{0}<c_{1}$ for some $c_{1}$, (see Figure 2 in Section 5)

- both of the above if $x_{0}=c_{1}$ (see Figure 3 in Section 5) or

- only the meridian of (i) if $c_{1}<x_{0}$, (see Figure 4 in Section 5)

at their other endpoint.

2. Preliminaries. We refer to [11] for basic tools in Riemannian geometry and [17] for some properties of geodesics on a surface of revolution.

Let $T^{2}:=\left(S^{1} \times S^{1}, d t^{2}+m(t)^{2} d \theta^{2}\right)$ be the torus defined in the introduction. The parameter curves $t=c$ and $\theta=c$ on the torus are called parallels and meridians respectively. By $(1.2)$, we get $m^{\prime}(n a)=0$ for any integer $n$. Thus the two parallels $t=0$ and $t=a$ are periodic geodesics on the torus $T^{2}$. The parallel $t=0$ (respectively $t=a$ ) is called the inner equator (respectively outer equator). Since the structure of the cut locus of a flat torus is well-known, we assume the Gaussian curvature of $T^{2}$ is non-zero hereafter. 
Lemma 2.1. The derivative function $m^{\prime}(t)$ of $m$ is positive on $(0, a)$.

Proof. Let $\mu:[0,2 a] \rightarrow T^{2}$ be a meridian emanating from a point $\mu(0)$ on $t=0$. We get by (1.4),

$$
m^{\prime \prime}(t)+K \circ \mu(t) m(t)=0 .
$$

Since $K \circ \mu$ is monotone non-decreasing on [0, $a$, it follows from (1.2) that $K \circ \mu$ attains a minimum at $t=0$ and a maximum at $t=a$. From the Gauss-Bonnet theorem, we have $\int_{0}^{2 a} K \circ \mu(t) m(t) d t=0$. Since $K \circ \mu$ is non-zero, the minimum $K \circ \mu(0)$ of $K \circ \mu$ is negative and the maximum $K \circ \mu(a)$ of $K \circ \mu$ is positive. From the intermediate value theorem and the monotonicity of $K \circ \mu$ on $[0, a]$ there exists a number $c \in(0, a)$ such that $K \circ \mu(t) \leq 0$ on $[0, c]$ and $K \circ \mu(t) \geq 0$ on $[c, a]$. Hence by (2.1), we have $m^{\prime \prime}(t) \geq 0$ on $[0, c], m^{\prime \prime}(t) \leq 0$ on $[c, a]$ and moreover $m^{\prime \prime}(0)>0, m^{\prime \prime}(a)<0$. This implies that $0=m^{\prime}(0)<m^{\prime}(t)$ for any $t \in(0, c]$ and $m^{\prime}(t)>m^{\prime}(a)=0$ for any $t \in[c, a)$. Therefore $m^{\prime}(t)$ is positive on $(0, a)$.

Let $\widetilde{M}:=\left(\mathbf{R}^{2}, d x^{2}+m(x)^{2} d y^{2}\right)$ denote the universal Riemannian covering manifold of the torus $T^{2}$, where $(x, y)$ denotes the canonical coordinates of $\mathbf{R}^{2}$. Without loss of generality, we may assume that $t \circ \pi=x$, and $\theta \circ \pi=y$, where $\pi: \widetilde{M} \rightarrow T^{2}$ denotes the covering projection. For each real number $c \in \mathbf{R}$, the arc $x=c$ is called a parallel arc on $\widetilde{M}$ and the $\operatorname{arc} y=c$ is called a meridian, which is a geodesic. Since $m^{\prime}(n a)=0$ for each integer $n$, the parallel arc $x=n a$ is a geodesic. Let $\gamma(s)=(x(s), y(s))$ be a unit speed geodesic on $\widetilde{M}$. There exists a constant $\nu$ such that

$$
m(x(s))^{2} y^{\prime}(s)=m(x(s)) \cos \eta(s)=\nu
$$

holds for any $s$, where $\eta(s)$ denotes the angle made by $\dot{\gamma}(s):=d \gamma_{t}\left(\frac{\partial}{\partial s}\right)$ and $\left(\frac{\partial}{\partial y}\right)_{\gamma(s)}$. This relation is a well-known formula, which is called the Clairaut relation. The constant $\nu$ is called the Clairaut constant of $\gamma$. Since $g(\dot{\gamma}(s), \dot{\gamma}(s))=1$, we have by $(2.2)$,

$$
x^{\prime}(s)= \pm \frac{\sqrt{m(x(s))^{2}-\nu^{2}}}{m(x(s))} .
$$

In particular $x^{\prime}(s)=0$ if and only if $m(x(s))=|\nu|$. It follows from (2.2) and (2.3) that for a unit speed geodesic $\gamma(s)=(x(s), y(s)), s_{1} \leq s \leq s_{2}$, with the Clairaut constant $\nu$,

$$
y\left(s_{2}\right)-y\left(s_{1}\right)=\varepsilon\left(x^{\prime}(s)\right) \int_{x\left(s_{1}\right)}^{x\left(s_{2}\right)} \frac{\nu}{m(x) \sqrt{m(x)^{2}-\nu^{2}}} d x,
$$

holds if $x^{\prime}(s) \neq 0$ on $\left(s_{1}, s_{2}\right)$ and moreover the length $L(\gamma)$ of $\gamma$ equals

$$
L(\gamma)=\varepsilon\left(x^{\prime}(s)\right) \int_{x\left(s_{1}\right)}^{x\left(s_{2}\right)} \frac{m(x)}{\sqrt{m(x)^{2}-\nu^{2}}} d x
$$

if $x^{\prime}(s) \neq 0$ on $\left(s_{1}, s_{2}\right)$, where $\varepsilon\left(x^{\prime}(s)\right)$ denotes the sign of $x^{\prime}(s)$. Since

$$
\frac{m}{\sqrt{m^{2}-\nu^{2}}}=\frac{\sqrt{m^{2}-\nu^{2}}}{m}+\frac{\nu^{2}}{m \sqrt{m^{2}-\nu^{2}},}
$$


we have

$$
L(\gamma)=\varepsilon\left(x^{\prime}(s)\right) \int_{x\left(s_{1}\right)}^{x\left(s_{2}\right)} \frac{\sqrt{m(x)^{2}-\nu^{2}}}{m(x)} d x+\nu\left(y\left(s_{2}\right)-y\left(s_{1}\right)\right),
$$

if $x^{\prime}(s) \neq 0$ on $\left(s_{1}, s_{2}\right)$.

LEMMA 2.2. If $\gamma:\left[0, s_{1}\right] \rightarrow \widetilde{M}$ is a geodesic segment with $(x \circ \gamma)^{\prime}(s) \neq 0$ on $\left[0, s_{1}\right)$, then for each $t \in\left(0, s_{1}\right] \gamma(t)$ is not conjugate to $\gamma(0)$ along $\gamma$. Hence if the absolute value of the Clairaut constant $\nu$ of a unit speed geodesic segment $\alpha$ is not greater than $m(0)$, then $\alpha$ has no conjugate point of $\alpha(0)$.

Proof. The first claim is clear from the equation (1.12) in [17], where Jacobi fields along a geodesic on a surface of revolution were computed. Suppose that $(x \circ \alpha)^{\prime}\left(s_{0}\right)=$ 0 for some $s_{0}$. By $(2.3), m\left(x\left(s_{0}\right)\right)=|\nu|=m(0)$. Thus, by Lemma 2.1, $x\left(s_{0}\right)=2 n a$ for some integer $n$ and $\alpha$ is tangent to the parallel arc $x=2 n a$, which is a geodesic. Therefore $\alpha$ must coincide with $x=2 n a$. Since the Gaussian curvature is negative on $x=2 n a, \alpha$ has no conjugate point of $\alpha(0)$.

For each $\nu \in[0, m(a)]$ let $\gamma_{\nu}(s)$ denote the unit speed geodesic emanating from $(a, 0)=\gamma_{\nu}(0)$ such that $\left(x \circ \gamma_{\nu}\right)^{\prime}(0) \leq 0$ and the angle $\angle\left(\dot{\gamma}_{\nu}(0),\left(\frac{\partial}{\partial y}\right)_{(a, 0)}\right)$ made by $\dot{\gamma}_{\nu}(0)$ and $\left(\frac{\partial}{\partial y}\right)_{(a, 0)}$ is $\arccos \frac{\nu}{m(a)}$. If $\nu \in(m(0), m(a))$, then $\gamma_{\nu}$ is tangent to the parallel arc $x=\xi(\nu)$ at a point $\gamma_{\nu}\left(t_{1}(\nu)\right)$, where $\xi(\nu)$ denotes the inverse function of $m \mid(0, a)$, and intersects the geodesic $x=a$ again at $\gamma_{\nu}\left(t_{0}(\nu)\right)$, where

$$
t_{0}(\nu):=\min \left\{t>0 ; x\left(\gamma_{\nu}(t)\right)=a\right\} .
$$

By (2.4), we get

$$
y\left(\gamma_{\nu}\left(t_{0}(\nu)\right)\right)=2\left(y\left(\gamma_{\nu}\left(t_{1}(\nu)\right)\right)-y\left(\gamma_{\nu}(0)\right)\right)=\varphi(\nu),
$$

where $\varphi:(m(0), m(a)) \rightarrow \mathbf{R}$ is the function defined by

$$
\varphi(\nu):=2 \int_{\xi(\nu)}^{a} \frac{\nu}{m(t) \sqrt{m(t)^{2}-\nu^{2}}} d t .
$$

Lemma 2.3. Let $\nu \in(m(0), m(a))$. For any $t \in \mathbf{R}$,

$$
\gamma_{\nu}\left(t+t_{0}(\nu)\right)=T_{\nu}\left(\gamma_{\nu}(t)\right)
$$

holds, where $T_{\nu}(x, y):=(2 a-x, y+\varphi(\nu))$.

Proof. Since $T_{\nu}$ is an isometry on $\widetilde{M}$ by $(1.2), \bar{\gamma}_{\nu}(t):=T_{\nu}\left(\gamma_{\nu}(t)\right)$ is a geodesic on $\widetilde{M}$. It is clear that $\bar{\gamma}_{\nu}(0)=\gamma_{\nu}\left(t_{0}(\nu)\right)$ and $\dot{\gamma}_{\nu}(0)=\dot{\gamma}_{\nu}\left(t_{0}(\nu)\right)$. Thus, by the uniqueness, $\bar{\gamma}_{\nu}(t)=\gamma_{\nu}\left(t+t_{0}(\nu)\right)$ for any real number $t$. Therefore the equation above is clear.

Lemma 2.4. Let $q_{1}=\left(c, y_{1}\right)$ and $q_{2}=\left(c, y_{2}\right)$ be two points on a parallel arc $x=c$. If $y_{1}>y_{2}>0$, then $d\left(q, q_{1}\right)>d\left(q, q_{2}\right)$ for any point $q$ on the meridian $y=0$, where $d$ denotes the Riemannian distance function of $\widetilde{M}$.

Proof. Let $q(t):=(c, t)$ be a point on the parallel arc $x=c$. For each $t>0$, let $\alpha_{t}:[0, d(q, q(t))] \rightarrow \widetilde{M}$ be a unit speed minimal geodesic segment joining $q$ to $q(t)$. Since the angle made by $\dot{\alpha}_{t}(d(q, q(t)))$ and $\left(\frac{\partial}{\partial y}\right)_{\alpha_{t}(d(q, q(t)))}$ is less $\frac{\pi}{2}$ by $(2.2)$, it follows from the first variational formula that $d(q, q(t))$ is strictly monotone increasing on $[0, \infty)$. Thus the claim of Lemma 2.4 is clear. 


\section{The cut loci of the torus and its universal covering manifold.}

Lemma 3.1. There is no cut point of the point $(a, 0)$ on $\widetilde{M}$ in the domain $(0, a) \times$ $(0, \infty)$.

Proof. Suppose that there exists a cut point of $p:=(a, 0)$ in the domain $(0, a) \times$ $(0, \infty)$. Since $\mathbf{R}^{\mathbf{2}}$ is simply connected, the cut locus $C_{p}$ of $p$ is a tree, i.e., a local tree which does not contain a circle. Hence this tree has an endpoint $q$, which is conjugate to $p$ along any minimal geodesic segment joining $p$ to $q$. Let $\gamma:[0, d(p, q)] \rightarrow \widetilde{M}$ denote a unit speed minimal geodesic segment joining $p=\gamma(0)$ to $q$. Let $c:[0, \delta] \rightarrow C_{p}$ be a unit speed rectifiable Jordan arc emanating from $q=c(0)$ (see [5] or [13]). Choose a positive number $\delta_{0}<\delta$ such that $0<x\left(c\left(\delta_{0}\right)\right)<a$ and $c\left(\delta_{0}\right)$ is a normal cut point of $p$, i.e., $c\left(\delta_{0}\right)$ is not conjugate to $p$ along any minimal geodesic segment joining $p$ to $c\left(\delta_{0}\right)$ and there exist exactly two minimal unit speed geodesic segments $\alpha$ and $\beta$ joining $p=\alpha(0)=\beta(0)$ and $c\left(\delta_{0}\right)$ (see [4] or [12]). Thus the geodesic segments $\alpha$ and $\beta$ bound a relatively compact domain $D(\alpha, \beta)$. There exists an endpoint $q_{1}$ in $D(\alpha, \beta)$, since the cut locus $C_{p}$ of $p$ is a tree. Thus $q_{1}$ is conjugate to $p$ along any minimal geodesic segments joining $p$ to $q_{1}$. We do not know whether $D(\alpha, \beta)$ contains $\gamma(0, d(p, q)]$ or not, but if we consider that $\gamma$ is a minimal geodesic segment joining $p$ to $q_{1}$, then we may assume that $D(\alpha, \beta)$ contains $\gamma(0, d(p, q)]$. Since $\widetilde{M}$ is symmetric with respect to the parallel arc $x=a$, the geodesic segments $\alpha$ and $\beta$ joining $p$ to $c\left(\delta_{0}\right)$ do not meet the arc $x=a$ again. Thus $D(\alpha, \beta)$ is contained in the domain in $(0, a) \times(0, \infty)$. Since $d(p, c(t))$ is a Lipschitz function, it follows from the Rademacher theorem $([10])$ that the function is differentiable for almost all $t$ and

$$
d\left(p, c\left(\delta_{0}\right)\right)-d(p, q)=\int_{0}^{\delta_{0}} \frac{d}{d t} d(p, c(t)) d t
$$

holds. For each normal cut point $c(t)$ on the curve, there exists a pair of two minimal unit speed geodesic segments $\alpha_{t}, \beta_{t}$ joining $p$ to $c(t)$. The angle $\angle\left(\dot{\alpha}_{t}\left(d(p, c(t)), \dot{\beta}_{t}(d(p, c(t)))\right.\right.$ is less than $\pi$, since $\left(y \circ \alpha_{t}\right)^{\prime}(s)$ and $\left(y \circ \beta_{t}\right)^{\prime}(s)$ are always positive by (2.2). Furthermore, it follows from Proposition 5.6 in [4] that the curve $c$ bisects the angle $\angle\left(\dot{\alpha}_{t}\left(d(p, c(t)), \dot{\beta}_{t}(d(p, c(t)))\right.\right.$ at $c(t)$ for each normal cut point $c(t)$ and hence $\frac{d}{d t} d(p, c(t))$ is positive for any normal cut points $c(t)$. Therefore, by (3.1), we get $d\left(p, c\left(\delta_{0}\right)\right)>d(p, q)$, i.e., $\gamma$ is shorter than $\beta$. Notice that $c(t)$ is a normal cut point for almost all $t$ (see [4], [6] or [18]). Since the geodesic segment $\gamma$ lies in the domain $D(\alpha, \beta)$, without loss of generality, we may assume that

$$
\angle\left(\dot{\beta}(0),\left(\frac{\partial}{\partial y}\right)_{p}\right)<\angle\left(\dot{\gamma}(0),\left(\frac{\partial}{\partial y}\right)_{p}\right)<\angle\left(\dot{\alpha}(0),\left(\frac{\partial}{\partial y}\right)_{p}\right) .
$$

Thus the number $s_{0}:=\max \{s \in[0, L(\gamma)] ; x(\gamma(t)) \leq x(\beta(t))$ for any $t \in[0, s]\}$ is positive. By supposing $s_{0}<L(\gamma)$, we will get a contradiction. Since $(x \circ \gamma)(t)-(x \circ$ $\beta)(t)$ is continuous,

$$
x\left(\gamma\left(s_{0}\right)\right)=x\left(\beta\left(s_{0}\right)\right) .
$$

Hence, by Lemma 2.4, we have

$$
y\left(\gamma\left(s_{0}\right)\right)=y\left(\beta\left(s_{0}\right)\right),
$$

since $d\left(p, \gamma\left(s_{0}\right)\right)=d\left(p, \beta\left(s_{0}\right)\right)=s_{0}$. By (3.3) and (3.4), we get $\gamma\left(s_{0}\right)=\beta\left(s_{0}\right)$, which is impossible. Therefore $s_{0}=L(\gamma)$, i.e.,

$$
x(\gamma(s)) \leq x(\beta(s))
$$


for any $s \in[0, L(\gamma)]$. It follows from (1.3), (1.4) and (3.5) that

$$
G(\gamma(s)) \leq G(\beta(s))
$$

for any $s \in[0, L(\gamma)]$. Here $G$ denotes the Gaussian curvature of $\widetilde{M}$. Thus, by the Rauch comparison theorem or the Sturm comparison theorem, there exists a conjugate point $\beta\left(t_{1}\right), t_{1} \in(0, L(\gamma)]$, of $p$ along $\beta$. This contradicts the minimality of $\beta$. Therefore there is no cut point of $p$ in the domain $(0, a) \times(0, \infty)$.

Proposition 3.1. The function $\varphi$, which we defined in (2.8), is monotone decreasing on $(m(0), m(a))$ and strictly monotone decreasing on the interval $\left(m(0), \nu_{0}\right)$ if $\varphi\left(\nu_{0}\right)>\varphi(m(a)):=\lim _{\nu \rightarrow m(a)}-\varphi(\nu)$.

Proof. Choose any two numbers $\nu_{1}<\nu_{2}$ from the interval $(m(0), m(a))$. Since $\arccos \frac{\nu_{2}}{m(a)}<\arccos \frac{\nu_{1}}{m(a)}$, it follows from Lemma 3.1 and $(2.2)$ that the geodesic segment $\left.\gamma_{\nu_{1}}\right|_{\left[0, t_{0}\left(\nu_{1}\right)\right]}$ does not enter the domain bounded by $\gamma_{\nu_{2}}\left[0, t_{0}\left(\nu_{2}\right)\right]$ and the geodesic segment $\{a\} \times\left[0, y\left(\gamma_{\nu_{2}}\left(t_{0}\left(\nu_{2}\right)\right)\right)\right]$. Thus $\varphi\left(\nu_{1}\right) \geq \varphi\left(\nu_{2}\right)$ by (2.7). Therefore there exists a unique limit $\varphi(m(a)):=\lim _{\nu \rightarrow m(a)}-\varphi(\nu)$. Furthermore suppose that $\varphi\left(\nu_{1}\right)=\varphi\left(\nu_{2}\right)$. This means the two geodesics $\gamma_{\nu_{1}}$ and $\gamma_{\nu_{2}}$ meet at $q_{0}:=\gamma_{\nu_{1}}\left(t_{0}\left(\nu_{1}\right)\right)$. From Lemma 3.1, for each $\nu \in\left[\nu_{1}, \nu_{2}\right]$, the geodesic $\gamma_{\nu}$ passes through the point $q_{0}$. This implies that $q_{0}$ is a conjugate point of the point $(a, 0)$ along $\gamma_{\nu}$ for each $\nu \in\left[\nu_{1}, \nu_{2}\right]$. By repeating the proof of the equation (3.5) for the pair $\gamma_{\nu_{1}}$ and $\gamma_{\nu_{2}}$, we get the inequality $x\left(\gamma_{\nu_{1}}(t)\right)<x\left(\gamma_{\nu_{2}}(t)\right)$ for any $t \in\left(0, t_{0}\left(\nu_{1}\right)\right)$. Thus by (1.3) and (1.4), $G\left(\gamma_{\nu_{1}}(t)\right) \leq G\left(\gamma_{\nu_{2}}(t)\right)$ for any $t \in\left[0, t_{0}\left(\nu_{1}\right)\right]$. Here $G$ denotes the Gaussian curvature of $\widetilde{M}$. Since the point $q_{0}$ is a conjugate point of $(a, 0)$ along $\gamma_{\nu}$ for each $\nu \in\left[\nu_{1}, \nu_{2}\right]$, it follows from the Rauch comparison theorem that $G\left(\gamma_{\nu_{1}}(t)\right)=G\left(\gamma_{\nu_{2}}(t)\right)$ for any $t \in\left[0, t_{0}\left(\nu_{1}\right)\right]$. Thus from (1.3) and (1.4), $G$ is constant on $x^{-1}\left(I_{t}\right)$, where $I_{t}:=\left[x\left(\gamma_{\nu_{1}}(t)\right), x\left(\gamma_{\nu_{2}}(t)\right)\right]$ for each $t \in\left(0, t_{0}\left(\nu_{1}\right)\right)$. Choose any $t_{1} \in\left(0, t_{0}\left(\nu_{1}\right)\right)$. Since $x\left(\gamma_{\nu_{1}}(t)\right)$ and $x\left(\gamma_{\nu_{2}}(t)\right)$ are continuous at $t=t_{1}, I_{t} \cap I_{t_{1}}$ is non-empty for any $t$, which is sufficiently close to $t_{1}$. Therefore $G$ is constant on $x^{-1}\left[\xi\left(\nu_{1}\right), a\right]$. Notice that $\min \left\{x\left(\gamma_{\nu_{1}}(t)\right) ; t \in\left[0, t_{0}\left(\nu_{1}\right)\right]\right\}=\xi\left(\nu_{1}\right)$ by $(2.3)$. This implies $\varphi$ is constant on $\left[\nu_{1}, m(a)\right]$, and hence $\varphi\left(\nu_{1}\right)=\varphi(m(a))$. Thus we have proved that $\varphi$ is strictly monotone decreasing on $\left(m(0), \nu_{0}\right)$ for any $\nu_{0} \in(m(0), m(a))$ with $\varphi\left(\nu_{0}\right)>\varphi(m(a))$. $\mathbf{\square}$

Proposition 3.2. The cut locus of the point $(a, 0)$ is $\{a\} \times([\varphi(m(a)), \infty)) \cup$ $(-\infty,-\varphi(m(a))])$.

Proof. Since $\widetilde{M}$ is symmetric with respect to $x=a$ and meridian $y=0$ respectively by (1.2), it follows from Lemma 3.1 that there is no cut point of $(a, 0)$ in $(0, a) \times(\mathbf{R} \backslash 0) \cup(a, 2 a) \times(\mathbf{R} \backslash 0)$. Since $\gamma_{m(0)}$ is obtained as the limit geodesic of minimal geodesic segments $\left.\gamma_{\nu}\right|_{\left[0, t_{0}(\nu)\right]}$ as $\nu$ goes to $m(0), \gamma_{m(0)}$ is a ray, i.e., any subarc of $\gamma_{m(0)}$ is a minimizing arc joining their endpoints. Hence $\gamma_{m(0)}$ has no cut point of the point $(a, 0)$. We will prove that there is no cut point of $(a, 0)$ in the unbounded domain $D$ bounded by $\gamma_{m(0)}$ and $\gamma_{m(0)}^{-}$containing the origin $(0,0)$, where $\gamma_{m(0)}^{-}$denotes the ray emanating from $(a, 0)$ which is reflectionally symmetric to $\gamma_{m(0)}$ with respect to $y=0$. Let $\gamma$ be a unit speed geodesic segment emanating from $(a, 0)$ to a point $q$ in the the domain $D$. Since $\gamma$ does not meet $\gamma_{m(0)}$ or $\gamma_{m(0)}^{-}$except $(a, 0)$, it follows from (2.2) that the absolute value of the Clairaut constant of $\gamma$ is not greater than $m(0)$. From Lemma 2.2 there is no conjugate point of $(a, 0)$ along $\gamma$. Thus, the cut locus of $(a, 0)$ is a subset of the parallel arc $x=a$. Since $\widetilde{M}$ is symmetric with respect to $x=a$, for each $\nu \in(m(0), m(a))$ the point $(a, \varphi(\nu))$ is joined by two minimal geodesic 
segments, which are symmetric with respect to $x=a$. Thus the point $(a, \varphi(\nu))$ is a cut point of $(a, 0)$ for each $\nu \in(m(0), m(a))$, i.e., $\{a\} \times[\varphi(m(a)), \infty)$ is a subset of the cut locus $C_{p}$ of $p$. Moreover it is clear that the point $(a, \varphi(m(a)))$ is an endpoint of the cut locus, since the geodesic segment $\gamma_{\nu}\left[0, t_{0}(\nu)\right]$ converges to the subarc of $x=a$ as $\nu$ tends to $m(a)$. Since $\widetilde{M}$ is symmetric with respect to $y=0$, the cut locus of $(a, 0)$ is $\{a\} \times([\varphi(m(a)), \infty) \cup(-\infty,-\varphi(m(a))])$.

Proposition 3.3. The cut locus of the origin $\tilde{p}(0):=(0,0)$ is empty. Moreover for each $u \in(0, a)$, the cut locus of $\tilde{p}(u):=(u, 0)$ is $\{2 a-u\} \times((-\infty,-\varphi(m(u))] \cup$ $[\varphi(m(u)), \infty))$. Hence the cut locus of $\tilde{p}(u+2 a)$ is $\{4 a-u\} \times((-\infty,-\varphi(m(u))] \cup$ $[\varphi(m(u)), \infty))$.

Proof. By (2.2), the absolute value of the Clairaut constant of any unit speed geodesic emanating from $\tilde{p}(0)$ is not greater than $m(0)$. It follows from Lemma 2.2 that there does not exist a conjugate point of $\tilde{p}(0)$ along any geodesic emanating from the point. Thus the cut locus of the point $\tilde{p}(0)$ is empty. Let a number $u \in(0, a)$ be fixed. For each $\nu \in[m(0), m(u))$, let $\alpha_{\nu}, \beta_{\nu}:[0, \infty) \rightarrow \widetilde{M}$ be two unit speed geodesics emanating from $\tilde{p}(u)$ such that

$$
\begin{gathered}
\angle\left(\dot{\alpha}_{\nu}(0),\left(\frac{\partial}{\partial y}\right)_{\tilde{p}(u)}\right)=\angle\left(\dot{\beta}_{\nu}(0),\left(\frac{\partial}{\partial y}\right)_{\tilde{p}(u)}\right)=\arccos \frac{\nu}{m(u)}, \\
\left(x \circ \alpha_{\nu}\right)^{\prime}(0)<0 \text { and }\left(x \circ \beta_{\nu}\right)^{\prime}(0)>0 .
\end{gathered}
$$

Let $\nu \in(m(0), m(u))$ be fixed and $t_{1}$ a parameter value of $\gamma_{\nu}$ satisfying $\left(x \circ \gamma_{\nu}\right)\left(t_{1}\right)=u$, and $\left(x \circ \gamma_{\nu}\right)^{\prime}\left(t_{1}\right)<0$. Since the geodesic defined by $\bar{\gamma}(t):=\gamma_{\nu}\left(t+t_{1}\right)-\left(0, y\left(\gamma_{\nu}\left(t_{1}\right)\right)\right)$ satisfies

$$
\bar{\gamma}(0)=\alpha_{\nu}(0), \quad \dot{\bar{\gamma}}(0)=\dot{\alpha}_{\nu}(0)
$$

we have

$$
\gamma_{\nu}\left(t+t_{1}\right)-\left(0, y\left(\gamma_{\nu}\left(t_{1}\right)\right)\right)=\bar{\gamma}(t)=\alpha_{\nu}(t)
$$

by the uniqueness. From Lemma 2.3, it is clear that

$$
(2 a-u, \varphi(\nu))=T_{\nu}\left(\alpha_{\nu}(0)\right)=\alpha_{\nu}\left(t_{0}(\nu)\right) .
$$

Similarly we get

$$
(2 a-u, \varphi(\nu))=T_{\nu}\left(\beta_{\nu}(0)\right)=\beta_{\nu}\left(t_{0}(\nu)\right) .
$$

By (3.7) and (3.8), both geodesics $\alpha_{\nu}$ and $\beta_{\nu}$ meet at the same point $(2 a-u, \varphi(\nu))$ again. For any point $q:=(2 a-u, y)$ on $\{2 a-u\} \times(\varphi(m(u)), \infty)$ there exist at most two geodesic segments $\left.\alpha_{\nu_{1}}\right|_{[0, d(\tilde{p}(u), q)]},\left.\beta_{\nu_{1}}\right|_{[0, d(\tilde{p}(u), q)]}$, where $\nu_{1}=\varphi^{-1}(y)$, joining $\tilde{p}(u)$ and $q$, which have the same length $t_{0}\left(\nu_{1}\right)$ by Proposition 3.1, (3.7) and (3.8). Thus these two geodesic segments must be minimal. Therefore any point $q$ on $\{2 a-u\} \times$ $[\varphi(m(u)), \infty)$ is a cut point of $\tilde{p}(u)$. Since $\left.\alpha_{\nu}\right|_{[0, d(\tilde{p}(u), q]}$ and $\left.\beta_{\nu}\right|_{[0, d(\tilde{p}(u), q)]}$ converge to $\alpha_{m(0)}$ and $\beta_{m(0)}$ respectively as $\nu \in(m(0), m(a))$ tends to $m(0)$, they are rays. Here a geodesic $\gamma:[0, \infty) \rightarrow \widetilde{M}$ is called a ray if any subarc of $\gamma$ is a minimal arc joining both endpoints. Then the two rays bound an unbounded domain $D_{1}$ 
containing $\{2 a-u\} \times[\varphi(m(u)), \infty)$. It is trivial that there is no cut point of $\tilde{p}(u)$ in $D_{1} \backslash\{2 a-u\} \times[\varphi(m(u)), \infty)$. Suppose that there exists a cut point $q$ of $\tilde{p}(u)$ in $\left\{p \in \widetilde{M} ; y(p) \geq 0, p \notin D_{1}\right\}$. By Lemma 2.2 and (2.2), the point $q$ is not conjugate along any minimal segment joining $\tilde{p}(u)$ to $q$. Thus, there exists a disc domain $D$ bounded by two minimal geodesic segments $\alpha, \beta$ joining $\tilde{p}(u)$ to $q$. The domain $D$ has a cut point $q_{0}$ of $\tilde{p}(u)$, which is an endpoint of the cut locus. The point $q_{0}$ is a conjugate point of $\tilde{p}(u)$ along any minimal geodesic segment joining $\tilde{p}(u)$ to $q_{0}$. By Lemma 2.2 and $(2.2), q_{0}$ is not conjugate to $\tilde{p}(u)$ along any minimal geodesic segments joining these two points. This is a contradiction. Thus, the cut locus of $\tilde{p}(u)$ in $\{p \in \widetilde{M} ; y(p) \geq 0\}$ is $\{2 a-u\} \times[\varphi(m(u)), \infty)$. Since $\widetilde{M}$ is symmetric with respect to the meridian $y=0$, the cut locus of $\tilde{p}(u)$ is $\{2 a-u\} \times((-\infty,-\varphi(m(u))] \cup[\varphi(m(u)), \infty)$. The last claim is clear from (1.2).

Let $\widetilde{E}(u)$ denote the set

$$
\widetilde{E}(u):=\{q \in \widetilde{M} ; d(\tilde{p}(u), q)=d(\tilde{p}(u+2 a), q)\}
$$

for each $u \in[0, a]$. It is clear from $(1.2)$ that $\widetilde{E}(0)=\{a\} \times \mathbf{R}$ and $\widetilde{E}(a)=\{2 a\} \times \mathbf{R}$.

Since the proofs of our main theorems (Theorems 3.1 and 3.2) need many lemmas to prove, we now give an overview of the steps leading up to them. For each point $p$ on the torus $T^{2}$ defined in the introduction, it is clear from the symmetry of $T^{2}$ that the opposite meridian of $p$ is a subset of the cut locus of $p$. Moreover, from a topological point of view, the cut locus of the point $p$ contains a Jordan curve which is freely homotopic to each parallel. If $p=\pi(\tilde{p}(u))$ for some $u$, where $\pi$ denotes the covering projection from $\widetilde{M}$ onto $T^{2}$, then the Jordan arc in question is the closure of

$$
E(u):=\{\pi(q) ; q \in \tilde{E}(u),|y(q)|<b\} .
$$

The inner equator and the closure of $E(u)$ divide $T^{2}$ into two domains $T_{0}(u)$ and $T_{1}(u)$. The precise definitions of these domains will be stated later. The structure of the cut locus is determined in each domain in Lemmas 3.3 and 3.4. If $C_{\tilde{p}(u)}$ and $\widetilde{E}(u)$ have no common point in the fundamental domain $(0,2 a) \times(-b, b)$ for any $u \in(0, a)$, then $T^{2}$ is said to be of non-standard type, otherwise it is said to be of standard type. In Lemma 3.6, a necessary and sufficient condition for $T^{2}$ to be of standard type is stated and its proof is given. By making use of Lemma 3.6, it is proven in Theorem 4.1 that all standard tori in Euclidean space are of standard type. Lemma 3.7 is for describing the case where the cut locus of a point looks like Figure 4 in Section 5. Lemmas 3.8 and 3.9 concern the cases where the cut loci of a point look like Figures 1 and 2 in Section 5, respectively.

Lemma 3.2. For each $u \in(0, a), \widetilde{E}(u)$ is a continuous curve in $(a, 2 a) \times \mathbf{R}$ and each compact subarc of $\widetilde{E}(u)$ is a Jordan arc.

Proof. Let $u \in(0, a)$ and $y \in \mathbf{R}$ be fixed. We will prove $f(a)<0<f(2 a)$, where $f(t):=d(\tilde{p}(u),(t, y))-d(\tilde{p}(u+2 a),(t, y))$. Since the minimal geodesic joining $\tilde{p}(u+2 a)$ to $(a, y)$ intersects $x=2 a$ at a point $q_{1}$, it follows from (1.2) that $d(\tilde{p}(2 a+u),(a, y))$ equals the length of the broken geodesic combined by the minimal geodesic segment joining $\tilde{p}(2 a-u)$ to $q_{1}$ and the minimal one joining $q_{1}$ to $(a, y)$. Thus,

$$
d(\tilde{p}(u+2 a),(a, y))>d(\tilde{p}(2 a-u),(a, y)) .
$$


By (1.2),

$$
d(\tilde{p}(2 a-u),(a, y))=d(\tilde{p}(u),(a, y)) .
$$

Combining (3.9) and (3.10), we obtain $f(a)<0$. By the similar argument as above, we get $f(2 a)>0$. By Proposition 3.3, $f(t)$ is $C^{\infty}$ on $(a, 2 a-u) \cup(2 a-u, 2 a)$. It is sufficient to prove that $f^{\prime}(t)$ is positive on $(a, 2 a-u) \cup(2 a-u, 2 a)$, because by the intermediate value theorem, $\eta(y) \in(a, 2 a)$ is uniquely determined by the equation $f(\eta(y))=0$. Thus it is clear that any compact subarc of $\widetilde{E}(u)$ is a Jordan arc. If $y=0$, then it is trivial that $f^{\prime}(t)$ is positive on $(a, 2 a)$. Thus we may assume $y \neq 0$. Choose any $t \in(a, 2 a-u) \cup(2 a-u, 2 a)$. Let $\gamma_{t}$ be the unit speed minimal geodesic segment joining $\tilde{p}(u)=\gamma_{t}(0)$ to $(t, y)=\gamma_{t}\left(L\left(\gamma_{t}\right)\right)$. By $(2.2), \gamma_{t}$ intersects $\mathbf{R} \times\{y\}$ exactly once. Therefore we get the closed domain $F_{t}:=\bigcup_{0 \leq s \leq L\left(\gamma_{t}\right)}\left[x\left(\gamma_{t}(s)\right), \infty\right) \times\left\{y\left(\gamma_{t}(s)\right)\right\}$. It is clear that $\tilde{p}(2 a+u) \in F_{t}$. Let $\beta_{t}$ denote the minimal geodesic segment joining $\tilde{p}(2 a+u)$ to $(t, y)$. Since $\beta_{t}$ lies in $F_{t}$,

$$
\angle\left(-\dot{\gamma}_{t}\left(L\left(\gamma_{t}\right)\right),\left(\frac{\partial}{\partial x}\right)_{(t, y)}\right)>\angle\left(-\dot{\beta}_{t}\left(L\left(\beta_{t}\right)\right),\left(\frac{\partial}{\partial x}\right)_{(t, y)}\right) .
$$

Therefore, by the first variation formula, $f^{\prime}(t)$ is positive and hence $f$ is strictly monotone increasing on $(a, 2 a)$.

Remark. For each $u \in[0, a], \widetilde{E}(u)$ is piecewise $C^{1}$.

By Lemma $3.2, \widetilde{E}(u), u \in[0, a)$ divides the open rectangle $(0,2 a) \times(-b, b)$ into two domains $\widetilde{T}_{0}(u)$ and $\widetilde{T}_{1}(u) \ni\left(\frac{a}{2}, 0\right)$.

Lemma 3.3. Let $u \in[0, a]$ be fixed. For each geodesic segment $\tilde{\alpha}:[0, l] \rightarrow \widetilde{M}$ emanating from $\tilde{p}(u)$ with $\tilde{\alpha}(0, l) \subset \widetilde{T}_{1}(u), \tilde{\alpha}$ is minimal if and only if $\alpha:=\pi \circ \tilde{\alpha}$ is minimal, where $\widetilde{T}_{1}(a):=(0,2 a) \times(-b, b)$. Hence $C_{p(u)} \cap \pi\left(\widetilde{T}_{1}(u)\right)=\pi\left(C_{\tilde{p}(u)} \cap \widetilde{T}_{1}(u)\right)$ for any $u \in[0, a]$. Here $p(u):=\pi(u, 0)$.

Proof. It is trivial that if $\alpha$ is minimal, then $\tilde{\alpha}$ is minimal. Thus, by supposing that $\tilde{\alpha}$ is minimal and $\alpha$ is not minimal, we will get a contradiction. Since $\alpha$ is not minimal, $\left.\alpha\right|_{\left[0, l_{0}\right]}$ is not minimal and $\tilde{\alpha}\left(0, l_{0}\right] \subset \widetilde{T}_{1}(u)$ for some $l_{0} \in(0, l)$. Thus, there exists a minimal geodesic segment $\beta:\left[0, l_{1}\right] \rightarrow T^{2}$ joining $p(u)$ to $\alpha\left(l_{0}\right)$, where $l_{1} \in(0, l)$. Let $\tilde{\beta}:\left[0, l_{1}\right] \rightarrow \widetilde{M}$ be the lifted geodesic segment of $\beta$, i.e., $\pi \circ \tilde{\beta}=\beta, \tilde{\beta}(0)=\tilde{p}(u)$. Since $\beta$ is minimal, $\tilde{\beta}$ is minimal and does not meet $\widetilde{E}(u)$. Thus $\tilde{\beta}\left(l_{1}\right)=\tilde{\alpha}\left(l_{0}\right)$, which contradicts the minimality of $\tilde{\alpha}$. The latter claim is a consequence of the first one.

It is trivial that any point on the meridian $\theta=b$ is a cut point of $p(u)=\pi(u, 0)$ for any $u \in[0,2 a]$. By Lemma 3.3, any point on the set

$$
E(u):=\{\pi(q) ; q \in \widetilde{E}(u),|y(q)|<b\}
$$

is also a cut point of $p(u)$ for each $u \in[0, a]$. Hence, for any $u \in[0, a]$, the cut locus of $p(u)$ contains $E(u) \cup\left\{q \in T^{2} ; \theta(q)=b\right\}$.

Definition 3.1. The torus $T^{2}$ is called of standard type if $C_{\tilde{p}(u)} \cap \widetilde{E}(u) \cap((0,2 a) \times$ $(-b, b))$ is non-empty for some $u \in[0, a]$.

Lemma 3.4. For each $u \in[0, a), C_{p(u)} \cap T_{0}(u)=\emptyset$, where $T_{0}(u):=\pi\left(\widetilde{T}_{0}(u)\right)$. Furthermore if $C_{\tilde{p}(u)} \cap \widetilde{E}(u)$ has an element $\left(2 a-u, y_{0}\right)$ in $(0,2 a) \times[-b, b]$ for some $u \in(0, a)$, then $C_{\tilde{p}(u)} \cap \widetilde{T}_{1}(u)=\{2 a-u\} \times\left(\left[\varphi(m(u)),\left|y_{0}\right|\right) \cup\left(-\left|y_{0}\right|,-\varphi(m(u))\right]\right)$. 
Proof. At first we prove $C_{p(u)} \cap T_{0}(u)=\emptyset$ for any $u \in[0, a)$. Suppose that there exists a cut point $\pi(q)$ of $p(u)$ in $T_{0}(u)$. Let $\alpha:[0, l] \rightarrow T^{2}$ be a minimal geodesic segment joining $p(u)$ to $\pi(q)$. Since $E(u)$ is a subset of the cut locus of $p(u), \alpha$ does not intersect $E(u)$. Since $\pi(q) \in T_{0}(u), \alpha$ intersects the inner equator. Thus $\tilde{\alpha}(l)$ is a point in $\widetilde{T}_{0}(u)$, where $\tilde{\alpha}:[0, l] \rightarrow \widetilde{M}$ denotes the lifted geodesic segment of $\alpha$ with $\tilde{\alpha}(0)=\tilde{p}(u+2 a)$. Since $\alpha(l)$ is not a conjugate point of $p(u)$ along $\alpha$ by Lemma 2.2 and (2.2), there exists another minimal geodesic segment $\beta$ joining $p(u)$ to $\pi(q)$. Then $\tilde{p}(u+2 a)$ and $\tilde{\alpha}(l)$ can be joined by two distinct minimal geodesic segments $\tilde{\alpha}$ and the lifted geodesic segment of $\beta$. Therefore $\tilde{\alpha}(l)$ is a cut point of $\tilde{p}(u+2 a)$. By Proposition 3.3 the point $\tilde{\alpha}(l)$ is not a cut point of $\tilde{p}(u+2 a)$. This is a contradiction. Thus, $C_{p(u)} \cap T_{0}(u)$ is empty. The second claim is clear from Proposition 3.3 and the following lemma.

Lemma 3.5. For each $u \in(0, a]$,

$$
\frac{d}{d t} d(\tilde{p}(u), \tilde{c}(t))>\frac{d}{d t} d(\tilde{p}(u+2 a), \tilde{c}(t))
$$

holds on $(\varphi(m(u)), \infty)$, where $\tilde{c}(t):=(2 a-u, t)$. Furthermore the function

$$
F(t, u):=d(\tilde{p}(u), \tilde{c}(t))-d(\tilde{p}(u+2 a), \tilde{c}(t))
$$

is $C^{1}$ on $\{(t, u) ; t>\varphi \circ m(u), u \in(0, a)\}$.

Proof. Choose any $u \in(0, a], t \in(\varphi(m(u)), \infty)$ and fix them. It follows from the proof of Proposition 3.3 that there exist exactly two unit speed minimal geodesic segments $\tilde{\alpha}_{\nu(t)}, \tilde{\beta}_{\nu(t)}:\left[0, l_{1}(t)\right] \rightarrow \widetilde{M}$ joining $\tilde{p}(u)$ to $\tilde{c}(t)$. Here $\nu(t):=\varphi^{-1}(t)$. It is clear that the domain $D_{t}$ bounded by these two minimal geodesic segments contains $\{2 a-u\} \times[\varphi(m(u)), t)$ and that any minimal geodesic segment joining $\tilde{p}(u+2 a)$ to $\tilde{c}(t)$ does not meet $D_{t}$. Thus we have

$$
\angle\left(-\dot{\tilde{c}}(t),-\dot{\tilde{\beta}}_{\nu(t)}\left(l_{1}(t)\right)\right)<\angle\left(-\dot{\tilde{c}}(t),-\dot{\tilde{\gamma}}_{t}\left(l_{0}(t)\right)\right)
$$

for each $t \in(\varphi(m(u)), \infty)$. Here $\tilde{\gamma}_{t}:\left[0, l_{0}(t)\right] \rightarrow \widetilde{M}$ denotes the minimal geodesic segment joining $\tilde{p}(u+2 a)$ to $\tilde{c}(t)$. Therefore it follows from the first variational formula that

$$
\frac{d}{d t} d(\tilde{p}(u), \tilde{c}(t))>\frac{d}{d t} d(\tilde{p}(u+2 a), \tilde{c}(t))
$$

holds on $(\varphi(m(u)), \infty)$. It follows from (1.2), (2.4) and (2.5) that for any $u \in(0, a)$ and $t>\varphi \circ m(u), d(\tilde{p}(u), \tilde{c}(t))=d(\tilde{p}(a),(a, t))$ holds. By Proposition 3.1, the function $\nu(t)=\varphi^{-1}(t)$ is continuous on $(\varphi \circ m(a), \infty)$. Thus the angle $\theta(t):=$ $\angle\left(\dot{\gamma}_{\nu(t)}\left(l_{t}\right),\left(\frac{\partial}{\partial y}\right)_{(a, t)}\right)$ is continuous on $(\varphi \circ m(a), \infty)$, where $l_{t}:=d(\tilde{p}(a),(a, t))$. It follows from the first variational formula (see Lemma 2.1 in [7]) that

$$
\frac{d}{d t} d(\tilde{p}(a),(a, t))=\cos \theta(t) .
$$

Thus $d(\tilde{p}(u), \tilde{c}(t))=d(\tilde{p}(a),(a, t))$ is $C^{1}$ on $\{(t, u) ; t>\varphi \circ m(u), u \in(0, a)\}$. It is clear from Proposition 3.2 that the function $d(\tilde{p}(u+2 a), \tilde{c}(t))$ is $C^{\infty}$ on $\{(t, u) ; t>$ $\varphi \circ m(u), u \in(0, a)\}$. Therefore the function $F$ is $C^{1}$.

By Lemmas 3.3, 3.4 and Proposition 3.3, we have 
Theorem 3.1. Let $T^{2}:=\left(S^{1} \times S^{1}, d t^{2}+m(t)^{2} d \theta^{2}\right)$ denote a torus with Riemannian metric $d t^{2}+m(t)^{2} d \theta^{2}$, where $d t^{2}$ and $d \theta^{2}$ denote the Riemannian metric of a circle with length $2 a>0,2 b>0$ respectively and $m$ is $a C^{\infty}$-function on $\mathbf{R}$ satisfying the equations (1.2) and (1.3). Then the cut locus $C_{p(u)}$ of $p(u):=\pi(u, 0)$ is $E(u) \cup\left\{q \in T^{2} ; \theta(q)=b\right\}$ for each $u \in\left[0, u_{0}\right]$, where $u_{0}:=\min \left(\{a\} \cup(\varphi \circ m)^{-1}(b)\right)$. Furthermore, if $T^{2}$ is of non-standard type, then $E(u) \cup\left\{q \in T^{2} ; \theta(q)=b\right\} \cup\{q \in$ $\left.\mathcal{P}_{u} ; \varphi(m(u)) \leq \theta(q) \leq 2 b-\varphi(m(u))\right\}$ is the cut locus of $p(u)$ for each $u \in\left(u_{0}, a\right]$. Here $\mathcal{P}_{u}$ denotes the parallel $t=2 a-u$.

REMARK. Suzuki proved in his thesis ([15]) that for any sufficiently small $u \in$ $[0, a]$, the cut locus of $p(u)$ is $E(u) \cup\left\{q \in T^{2} ; \theta(q)=b\right\}$. If we divide a standard torus by a finite group of rotations around the $z$-axis, we can get a torus with $\varphi(m(a))<b$, which is of non-standard type.

Let $B_{0}(u)$ (respectively $B_{1}(u)$ ) denote the distance between $\tilde{p}(u+2 a)$ (respectively $\tilde{p}(u))$ and $(2 a-u, b)$. Suppose $\varphi(m(a))<b$. By Lemma 2.1 and Proposition 3.1, there exists a unique solution $u=u_{*} \in(0, a)$ of $\varphi \circ m(u)=b$. It follows from (2.4) and (2.6) that

$$
B_{0}{ }^{\prime}(u)=2 \frac{\sqrt{m(u)^{2}-\nu_{0}^{2}}}{m(u)}, \quad B_{1}(u)=B_{1}(a)
$$

for any $u \in(0, a)$ and $u \in\left[u_{*}, a\right]$ respectively, where $\nu_{0} \in(0, m(0))$ is the number satisfying

$$
b=2 \int_{0}^{u} \frac{\nu_{0}}{m(t) \sqrt{m(t)^{2}-\nu_{0}^{2}}} d t .
$$

Lemma 3.6. The torus $T^{2}$ is of standard type if and only if $\varphi(m(a))<b$ and $B_{0}\left(u_{*}\right)<B_{1}\left(u_{*}\right)$.

Proof. Suppose $T^{2}$ is of standard type. Hence, by Proposition 3.3, there exist numbers $u_{0} \in(0, a), y_{0} \in(-b, b)$ such that $\left(2 a-u_{0}, y_{0}\right) \in C_{\tilde{p}\left(u_{0}\right)} \cap \widetilde{E}\left(u_{0}\right)$. It is clear that $\varphi\left(m\left(u_{0}\right)\right) \leq\left|y_{0}\right|<b$ by Proposition 3.3. Hence by Proposition 3.1 and Lemma 2.1 , we get $\varphi(m(a))<b$. Supposing $B_{0}\left(u_{*}\right) \geq B_{1}\left(u_{*}\right)$, we will get a contradiction. By (3.11), for any $u \in\left(u_{*}, a\right), B_{0}(u)>B_{0}\left(u_{*}\right) \geq B_{1}(u)$. Hence by Lemma 3.5, $C_{\tilde{p}(u)} \cap \widetilde{E}(u) \cap(0,2 a) \times(-b, b)$ is empty for any $u>u_{*}$. By Lemma 2.1 and Proposition $3.1, \varphi(m(u)) \geq \varphi\left(m\left(u_{*}\right)\right)=b$ for any $u \in\left[0, u_{*}\right]$. Thus, $C_{\tilde{p}(u)} \cap \widetilde{E}(u) \cap(0,2 a) \times(-b, b)$ is empty for any $u \in[0, a]$. This is a contradiction. Suppose $\varphi(m(a))<b$ and $B_{0}\left(u_{*}\right)<B_{1}\left(u_{*}\right)$. Since $\widetilde{M}$ is symmetric with respect to $x=2 a, B_{0}(a)$ equals the length of the broken geodesic of two minimal geodesics, the minimal geodesic joining $\tilde{p}(a)$ and $\left(2 a, \frac{b}{2}\right)$, and the one joining $\left(2 a, \frac{b}{2}\right)$ and $(a, b)$. Thus it is clear that $B_{1}(a)=$ $d(\tilde{p}(a),(a, b))<B_{0}(a)$. From the intermediate value theorem, it follows that there exists $u_{0} \in\left(u_{*}, a\right)$ such that $B_{0}\left(u_{0}\right)=B_{1}\left(u_{0}\right)$. By Proposition 3.1 and Lemma 2.1, $\varphi\left(m\left(u_{0}\right)\right)<\varphi\left(m\left(u_{*}\right)\right)=b$. Thus $C_{\tilde{p}\left(u_{0}\right)} \cap \widetilde{E}\left(u_{0}\right) \cap(0,2 a) \times(-b, b)$ has an element $\left(2 a-u_{0}, \varphi\left(m\left(u_{0}\right)\right)\right)$ Therefore $T^{2}$ is of standard type.

Lemma 3.7. If $T^{2}$ is of standard type, then there exists a unique solution $u=$ $u_{1} \in\left(u_{*}, a\right)$ of $B_{0}(u)=B_{1}(u)\left(=B_{1}\left(u_{*}\right)\right)$ such that for any $u \in\left(u_{1}, a\right]$,

$$
\left.\widetilde{T}_{1}(u) \cap C_{\tilde{p}(u)}=\{2 a-u\} \times([\varphi(m(u)), b) \cup(-b,-\varphi(m(u)))]\right) .
$$


Proof. By Lemma 3.6, $B_{0}\left(u_{*}\right)<B_{1}\left(u_{*}\right)$. As we observed in the proof of Lemma 3.6, $B_{1}(a)<B_{0}(a)$ holds. Hence it follows from the intermediate value theorem that there exists a solution $u=u_{1} \in\left(u_{*}, a\right)$ of $B_{0}(u)=B_{1}\left(u_{*}\right)=B_{1}(u)$. Choose any $u \in\left(u_{1}, a\right]$. By Lemma 2.1, Proposition 3.1 and $(3.11)$, we get $B_{0}(u)>B_{0}\left(u_{1}\right)=$ $B_{1}\left(u_{1}\right)=B_{1}(u)$ and $\varphi(m(u))<\varphi\left(m\left(u_{*}\right)\right)=b$. Therefore, by Proposition 3.3 and Lemma 3.5,

$$
C_{\tilde{p}(u)} \cap \widetilde{T}_{1}(u)=\{2 a-u\} \times([\varphi(m(u)), b) \cup(-b,-\varphi(m(u))]) .
$$

LEMMA 3.8. If $T^{2}$ is of standard type, then there exists a unique solution $u=$ $u_{2} \in\left(u_{*}, u_{1}\right)$ of $d(\tilde{p}(u+2 a), \tilde{\varphi}(u))=d(\tilde{p}(u), \tilde{\varphi}(u))$, where $\tilde{\varphi}(u):=(2 a-u, \varphi \circ m(u))$, such that for any $u \in\left[0, u_{2}\right]$,

$$
\widetilde{T}_{1}(u) \cap C_{\tilde{p}(u)}=\emptyset
$$

Proof. For simplicity, we set

$$
\Phi_{0}(u):=d(\tilde{p}(u+2 a), \tilde{\varphi}(u)), \quad \Phi_{1}(u):=d(\tilde{p}(u), \tilde{\varphi}(u)) .
$$

First, we will prove that $\Phi_{0}-\Phi_{1}$ is strictly monotone increasing on $\left(u_{*}, a\right)$. Choose any number $u \in\left(u_{*}, a\right)$ and fix it. By (1.2), (2.4) and (2.6),

$$
\begin{gathered}
\Phi_{0}(u)=2 \int_{0}^{u} \frac{\sqrt{m(t)^{2}-\nu_{0}^{2}}}{m(t)} d t+\nu_{0} \varphi(m(u)), \\
\Phi_{1}(u)=2 \int_{u}^{a} \frac{\sqrt{m(t)^{2}-m(u)^{2}}}{m(t)} d t+m(u) \varphi(m(u)),
\end{gathered}
$$

where $\nu_{0} \in(0, m(0))$ denotes the number (depending on $u$ ) such that

$$
\varphi(m(u))=2 \int_{0}^{u} \frac{\nu_{0}}{m(t) \sqrt{m(t)^{2}-\nu_{0}^{2}}} d t .
$$

Hence we have

$$
\Phi_{0}{ }^{\prime}(u)=f\left(\nu_{0}\right), \quad \Phi_{1}{ }^{\prime}(u)=f(m(u))
$$

where

$$
f(t):=2 \frac{\sqrt{m(u)^{2}-t^{2}}}{m(u)}+t(\varphi \circ m)^{\prime}(u), 0 \leq t \leq m(u) .
$$

From Proposition 3.1 and Lemma 2.1, the function $f$ is strictly monotone decreasing on $(0, m(u))$. Thus, we have $\Phi_{0}{ }^{\prime}(u)>\Phi_{1}{ }^{\prime}(u)$. This implies $\Phi_{0}-\Phi_{1}$ is strictly monotone increasing on $\left(u_{*}, a\right)$. By Lemmas 3.5 and 3.7 , we get

$$
0=B_{1}\left(u_{1}\right)-B_{0}\left(u_{1}\right)>\Phi_{1}\left(u_{1}\right)-\Phi_{0}\left(u_{1}\right) .
$$

Hence

$$
\Phi_{0}\left(u_{1}\right)>\Phi_{1}\left(u_{1}\right) .
$$


On the other hand, by Lemma 3.6,

$$
\Phi_{0}\left(u_{*}\right)=B_{0}\left(u_{*}\right)<B_{1}\left(u_{*}\right)=\Phi_{1}\left(u_{*}\right) .
$$

By (3.13) and (3.14), there exists a solution $u=u_{2} \in\left(u_{*}, u_{1}\right)$ of $\Phi_{0}(u)=\Phi_{1}(u)$. Choose any $u \in\left(u_{*}, u_{2}\right]$ and fix it. Since $\Phi_{0}-\Phi_{1}$ is strictly monotone increasing on $\left(u_{*}, a\right)$, we get $\left(\Phi_{0}-\Phi_{1}\right)(u) \leq\left(\Phi_{0}-\Phi_{1}\right)\left(u_{2}\right)=0$. Thus, $(2 a-u, \varphi(m(u))) \in \widetilde{T}_{0}(u)$. By Lemma 3.5, $C_{\tilde{p}(u)} \cap \widetilde{T}_{1}(u)=\emptyset$. On the other hand, for any $u \in\left[0, u_{*}\right], \varphi(m(u)) \geq b$ and hence

$$
C_{\tilde{p}(u)} \cap(0,2 a) \times(-b, b)=\emptyset .
$$

Therefore for any $u \in\left[0, u_{2}\right], C_{\tilde{p}(u)} \cap \widetilde{T}_{1}(u)$ is empty.

LEMma 3.9. If $T^{2}$ is of standard type, then there exists a $C^{1}$-function $b(u)$ on $\left(u_{2}, u_{1}\right)$ such that

$$
C_{\tilde{p}(u)} \cap \widetilde{T}_{1}(u)=\{2 a-u\} \times([\varphi(m(u)), b(u)) \cup(-b(u),-\varphi(m(u))])
$$

for each $u \in\left(u_{2}, u_{1}\right)$.

Proof. Since $\Phi_{0}-\Phi_{1}$ is strictly monotone increasing on $\left(u_{*}, a\right)$ as we observed in the proof of Lemma 3.8,

$$
\Phi_{0}(u)-\Phi_{1}(u)>\Phi_{0}\left(u_{2}\right)-\Phi_{1}\left(u_{2}\right)=0
$$

for any $u>u_{2}$. Thus,

$$
\Phi_{0}(u)>\Phi_{1}(u)
$$

for any $u \in\left(u_{2}, a\right)$. From (3.11), $B_{0}-B_{1}$ is strictly monotone increasing on $\left(u_{*}, a\right)$. Thus we get $0=B_{0}\left(u_{1}\right)-B_{1}\left(u_{1}\right)>B_{0}(u)-B_{1}(u)$, i.e.,

$$
B_{0}(u)<B_{1}(u)
$$

for any $u \in\left(u_{*}, u_{1}\right)$. Choose any $u \in\left(u_{2}, u_{1}\right)$ and fix it. By Lemma $3.5 \frac{\partial F}{\partial t}(t, u)$ is positive for any $t$ and $u \in(0, a)$ with $t>\varphi(m(u))$, where $F(t, u):=d(\tilde{p}(u+2 a),(2 a-$ $u, t))-d(\tilde{p}(u),(2 a-u, t))$. Furthermore we have $F(b, u)<0<F(\varphi(m(u)), u)$ by (3.15) and (3.16). From the intermediate value theorem, there exists a number $b(u) \in$ $(\varphi \circ m(u), b)$ such that $F(b(u), u)=0$. It follows from the implicit function theorem and Lemma 3.5 that the function $b(u)$ is $C^{1}$ on $\left(u_{2}, u_{1}\right)$. Since $(2 a-u, b(u)) \in \widetilde{E}(u) \cap C_{\tilde{p}(u)}$ for each $u \in\left(u_{2}, u_{1}\right)$, it follows from Lemma 3.4 that

$$
C_{\tilde{p}(u)} \cap \widetilde{T}_{1}(u)=\{2 a-u\} \times([\varphi(m(u)), b(u)) \cup(-b(u),-\varphi(m(u))])
$$

for each $u \in\left(u_{2}, u_{1}\right)$.

By Lemmas 3.3, 3.4, 3.7, 3.8 and 3.9, we have,

TheOrem 3.2. Let $T^{2}:=\left(S^{1} \times S^{1}, d t^{2}+m(t)^{2} d \theta^{2}\right)$ denote a torus with Riemannian metric $d t^{2}+m(t)^{2} d \theta^{2}$, where $d t^{2}$ and $d \theta^{2}$ denote the Riemannian metric of a circle with length $2 a>0,2 b>0$ respectively and $m$ is a $C^{\infty}$-function on $\mathbf{R}$ satisfying the equations (1.2) and (1.3). If $T^{2}$ is of standard type, then the cut locus $C_{p(u)}$ of $p(u)$ has the following structure. 
(1) $C_{p(u)}=F(u)$ for each $u \in\left[0, u_{2}\right]$, where

$$
F(u):=E(u) \cup\left\{q \in T^{2} ; \theta(q)=b\right\} .
$$

(2) $C_{p(u)}=F(u) \cup\left\{q \in \mathcal{P}_{u} ; \varphi(m(u)) \leq \theta(q) \leq b(u)\right\} \cup\left\{q \in \mathcal{P}_{u} ; 2 b-b(u) \leq \theta(q) \leq\right.$ $2 b-\varphi(m(u))\}$ for each $u \in\left(u_{2}, u_{1}\right)$.

(3) $C_{p(u)}=F(u) \cup\left\{q \in \mathcal{P}_{u} ; \varphi(m(u)) \leq \theta(q) \leq 2 b-\varphi(m(u))\right\}$ for each $u \in\left[u_{1}, a\right]$. Here $\mathcal{P}_{u}:=\left\{q \in T^{2} ; t(q)=2 a-u\right\}$.

4. Tori of elliptical cross-section. Finally, we prove that each torus $T_{e}$ defined by

$$
\left(\sqrt{x^{2}+y^{2}}-R\right)^{2}+\left(\frac{z}{e}\right)^{2}=r^{2}(R>r>0,1 \geq e>0)
$$

is of standard type. Note that the Riemannian metric of the torus $T_{e}$ satisfies (1.3). It is clear that $T_{e}$ is isometric to the torus $\left(S^{1} \times S^{1}, d t^{2}+m(t)^{2} d \theta^{2}\right)$ with a warped product metric $d t^{2}+m(t)^{2} d \theta^{2}$, where $d t^{2}$ and $d \theta^{2}$ denote the Riemannian metric of a circle with length $2 a:=r \int_{0}^{2 \pi} \sqrt{\sin ^{2} \theta+e^{2} \cos ^{2} \theta} d \theta$ and $2 \pi$ respectively, and $m$ is the solution of the differential equation

$$
m^{\prime}(t)^{2}=\frac{r^{2}-(m(t)-R)^{2}}{r^{2}+\left(e^{2}-1\right)(m(t)-R)^{2}}
$$

with initial condition $m(0)=R-r$. It is easy to check that the function $m$ satisfies (1.2) and (1.3) for any constants $R>r>0, e \in(0,1]$.

Lemma 4.1. For each torus $T_{e}$ defined by (4.1), $\varphi \circ m\left(\frac{a}{2}\right)<\pi$. Hence the number $u_{*}$ is less than $\frac{a}{2}$.

Proof. By definition,

$$
\varphi \circ m\left(\frac{a}{2}\right)=2 \int_{\frac{a}{2}}^{a} \frac{m\left(\frac{a}{2}\right)}{m(t) \sqrt{m(t)^{2}-m\left(\frac{a}{2}\right)^{2}}} d t .
$$

Setting $x=\frac{1}{r}(m(t)-R)$, we get

$$
\varphi \circ m\left(\frac{a}{2}\right)=2 \sqrt{\xi} \int_{0}^{1} \frac{\sqrt{1+\left(e^{2}-1\right) x^{2}}}{(1+\xi x) \sqrt{x(1-x)} \sqrt{1+x} \sqrt{2+\xi x}} d x,
$$

where $\xi:=\frac{r}{R} \in(0,1)$. Note that $m\left(\frac{a}{2}\right)=R$ and $m(a)=R+r$. Since $\frac{1}{\sqrt{1+x}} \leq \frac{\sqrt{2}}{\sqrt{2+\xi x}}$ and $\sqrt{1+\left(e^{2}-1\right) x^{2}} \leq 1$

$$
\varphi \circ m\left(\frac{a}{2}\right) \leq 2 \sqrt{2 \xi} \int_{0}^{1} \frac{1}{(1+\xi x) \sqrt{x(1-x)}(2+\xi x)} d x .
$$

Since

$$
\begin{gathered}
\int \frac{d x}{(1+\xi x)(2+\xi x) \sqrt{x(1-x)}}= \\
\frac{-2}{\sqrt{1+\xi}} \tan ^{-1} \sqrt{\frac{1-x}{x(1+\xi)}}+\sqrt{\frac{2}{2+\xi}} \tan ^{-1} \sqrt{\frac{2(1-x)}{x(2+\xi)}}
\end{gathered}
$$


we have

$$
\int_{0}^{1} \frac{d x}{(1+\xi x)(2+\xi x) \sqrt{x(1-x)}}=\left(\frac{2}{\sqrt{1+\xi}}-\sqrt{\frac{2}{2+\xi}}\right) \frac{\pi}{2} .
$$

By (4.3) and (4.4), we get

$$
\varphi \circ m\left(\frac{a}{2}\right) \leq \sqrt{2 \xi}\left(\frac{2}{\sqrt{1+\xi}}-\sqrt{\frac{2}{2+\xi}}\right) \pi .
$$

Since the function $\sqrt{x}\left(\frac{2}{\sqrt{1+x}}-\sqrt{\frac{2}{2+x}}\right)$ is monotone increasing on $[0,1]$, we get

$$
\varphi \circ m\left(\frac{a}{2}\right)<2\left(1-\frac{1}{\sqrt{3}}\right) \pi<\pi .
$$

The latter claim follows from Proposition 3.1 and Lemma 2.1.

Theorem 4.1. Each torus $T_{e}$ defined by (4.1) is of standard type.

Proof. It follows from Lemmas 3.6, 4.1 and (3.11) that it is sufficient to prove that

$$
\left(B_{0}\left(u_{*}\right)<\right) B_{0}\left(\frac{a}{2}\right)<B_{1}\left(\frac{a}{2}\right)\left(=B_{1}\left(u_{*}\right)\right)
$$

By $(2.6)$,

$$
B_{1}\left(\frac{a}{2}\right)=2 \int_{u_{*}}^{a} \frac{\sqrt{m(t)^{2}-m\left(u_{*}\right)^{2}}}{m(t)} d t+\pi m\left(u_{*}\right) .
$$

Setting

$$
f(u):=2 \int_{u}^{a} \frac{\sqrt{m^{2}-m(u)^{2}}}{m} d t+\pi m(u), u \in[0, a]
$$

we have

$$
f^{\prime}(u)=-2 m^{\prime}(u) \int_{u}^{a} \frac{m(u)}{m \sqrt{m^{2}-m(u)^{2}}} d t+\pi m^{\prime}(u)=(-\varphi \circ m(u)+\pi) m^{\prime}(u) .
$$

Thus, by Lemma 2.1 and Proposition 3.1, $f$ attains a maximum at $u=u_{*}$. Hence, by (4.6),

$$
B_{1}\left(\frac{a}{2}\right)=f\left(u_{*}\right)>f\left(\frac{a}{2}\right)>\pi m\left(\frac{a}{2}\right)=\pi R .
$$

Since $B_{0}{ }^{\prime}(u)<2$ by $(3.11)$, it follows from the mean value theorem that

$$
B_{0}\left(\frac{a}{2}\right)-B_{0}(0)<a .
$$

Therefore

$$
B_{0}\left(\frac{a}{2}\right)<a+d(\tilde{p}(2 a),(2 a, \pi))=a+m(0) \pi \leq \pi R .
$$

By (4.7) and (4.8), we have $B_{0}\left(\frac{a}{2}\right)<B_{1}\left(\frac{a}{2}\right)$. 
5. Illustrations. We provide illustrations (Figures 1 to 4 ) for the case of the torus defined by (1.1) with $R=2$ and $r=1$, i.e. the surface given by

$$
\left(\sqrt{x^{2}+y^{2}}-2\right)^{2}+z^{2}=1
$$

for which we find that $u_{*} \approx 0.63514, u_{2} \approx 2.43309$ and $u_{1} \approx 2.98009$.

We have applied standard numerical and symbolic methods and the software tool Loki [14] to the methods of this paper. Loki computes cut loci from points on surfaces via an approximation to the exponential map by a piecewise polynomial defined upon the universal covering. Inverting this approximation to the exponential map gives the global information necessary to approximate cut points.

\section{REFERENCES}

[1] G. Buiss, The geodesic lines on the anchor ring, Ann. of Math., 4 (1903), pp. 1-21.

[2] D. ElerATh, An improved Toponogov comparison theorem for non-negatively curved manifolds, J. Differential Geom., 15 (1980), pp. 187-216.

[3] H. Gluck And D. Singer, Scattering of geodesic fields, II, Ann. of Math., 110 (1979), pp. 205-225.

[4] P. Hartman, Geodesic parallel coordinates in the large, Amer. J. Math., 86 (1964), pp. 705-727.

[5] J. J. HebDA, Metric structure of cut loci in surfaces and Ambrose's problem, J. of Differential Geom., 40 (1994), pp. 621-642.

[6] J. Iтон AND M. TANAKA, The Hausdorff dimension of a cut locus on a smooth Riemannian manifold, Tohoku Math. J., 50 (1998), pp. 571-575.

[7] J. Iтон And M. TanaKa, The Lipschitz continuity of the distance function to the cut locus, Trans. Amer. Math. Soc., 353 (2001), pp. 21-40.

[8] B. Kimball, Geodesics on a toroid, Amer. J. Math., 52 (1930), pp. 29-52.

$[9]$ H. VON MANGOLDT, Über diejenigen Punkte auf positiv gekrümmten Flächen, welche die Eingenschaft haben, dass die von Ihnen ausgehenden geodätischen Linien nie aufhören, kürzeste Linien zu sein, J. Reine. Angew. Math., 91 (1881), pp. 23-53.

[10] F. Morgan, Geometric measure theory, A beginner's guide, Acad. Press 1988.

[11] T. SAKAI, Riemannian Geometry, Mathematical Monograph, Amer. Math. Soc., 8 (1997)

[12] K. Shiohama and M. Tanaka, An isoperimetric problem for infinitely connected complete noncompact surfaces, Geometry of Manifolds, Perspectives in Math., Academic Press, Boston-San Diego-New York-Berkeley-London-Sydney-Tokyo-Tronto, 8 (1989), pp. 317343.

[13] K. Shiohama And M. Tanaka, Cut loci and distance spheres on Alexandrov surfaces, Séminaires \& Congrès, Collection SMF No.1, Actes de la table ronde de Géométrie différentielle en l'honneur Marcel Berger, (1996), pp. 531-560.

[14] R. Sinclair and M. Tanaka, Loki: Software for computing cut loci, Experimental Mathematics, 11 (2002), pp. 1-25.

[15] K. SuzukI, The cut locus of a Riemannian manifold, (in Japanese) Master thesis, Tokyo Institute of Technology (1997).

[16] M. Tanaka, On the cut loci of a von Mangoldt's surface of revolution, J. Math. Soc. Japan, 44 (1992), pp. 631-641.

[17] M. TANAKa, On a characterization of a surface of revolution with many poles, Memoirs Fac. Sci. Kyushu Univ. Ser. A, 46 (1992), pp. 251-268.

[18] M. TANAKa, Characterization of a differentiable point of the distance function to the cut locus, J. Math. Soc. Japan, 55 (2003), pp. 231-243. 


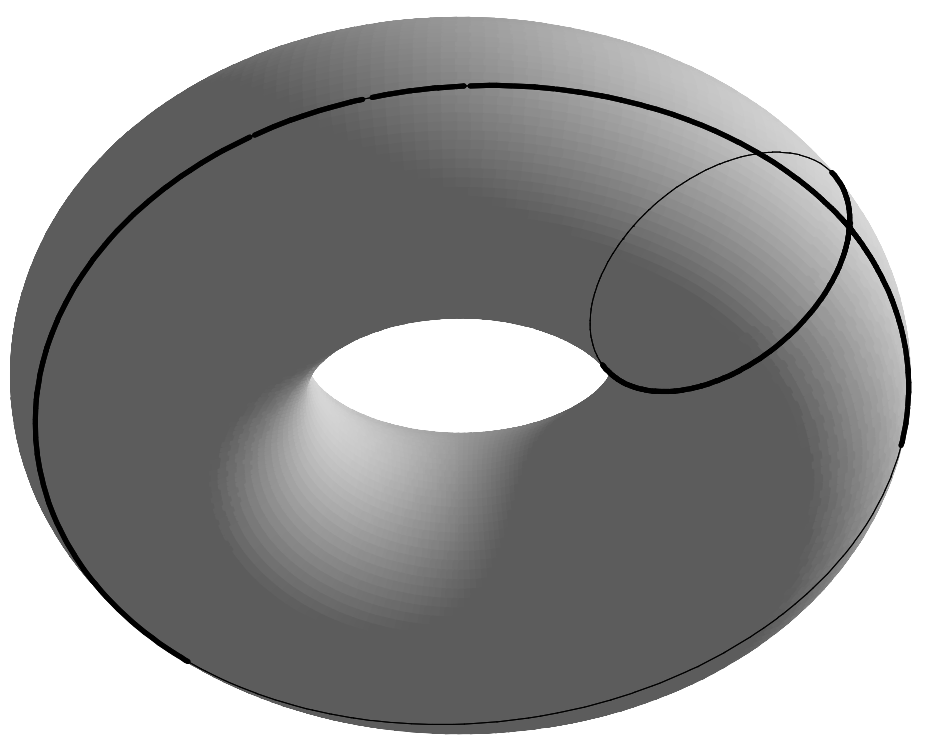

FIG. 1. $C_{p\left(u_{*}\right)}\left(u_{*}<u_{2}\right)$

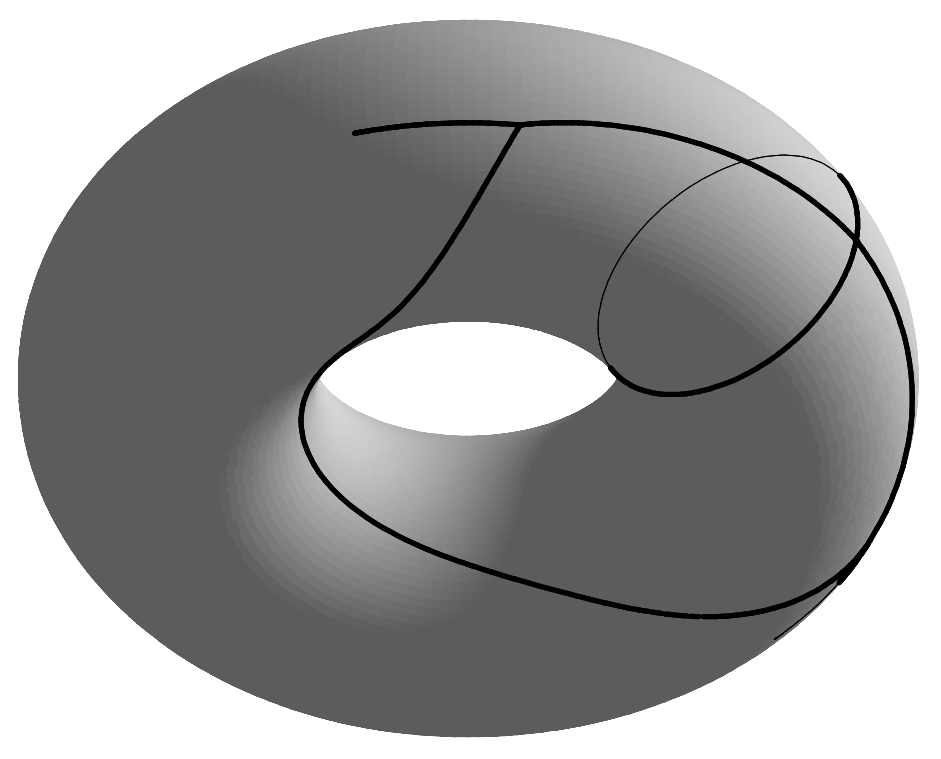

FIG. 2. $C_{p(2.7)}\left(u_{2}<2.7<u_{1}\right)$ 


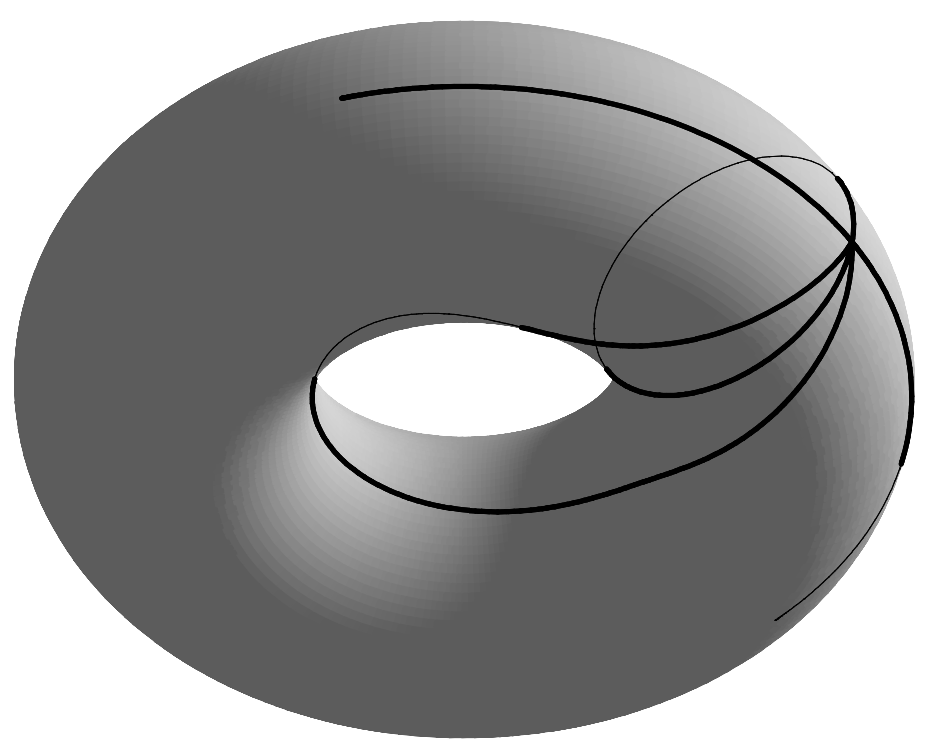

FIG. 3. $C_{p\left(u_{1}\right)}$

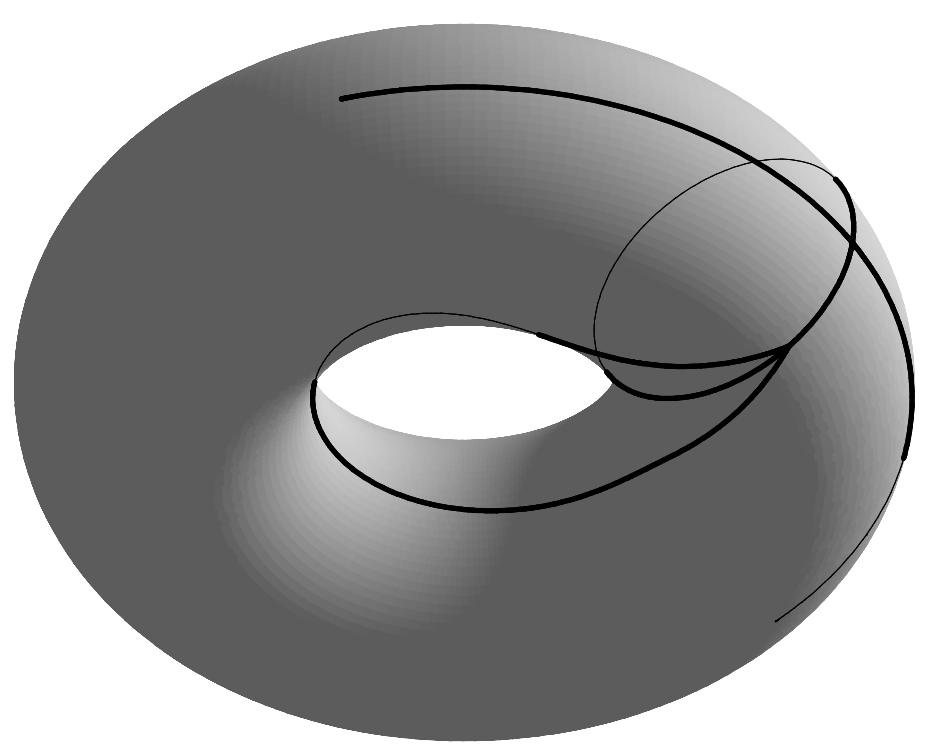

FIG. 4. $C_{p(3.0)}\left(u_{1}<3.0<a\right)$ 